\title{
Painful Oral Ulceration Developed as Lichenoid Contact Reaction. A Case Report
}

\author{
Glauco Vieira ${ }^{1}$, Margareth Oda ${ }^{1}$, Patrícia de Freitas ${ }^{1}$ and Dante Migliari ${ }^{2 *}$ \\ ${ }^{1}$ Department of Operative Dentistry, School of Dentistry, University of Sao Paulo, Brazil \\ ${ }^{2}$ Department of Stomatology, School of Dentistry, University of Sao Paulo, Brazil
}

Received: August 19, 2016; Accepted: August 26, 2016; Published: August 30, 2016

*Corresponding author: Dante A. Migliari, Universidade de São Paul, Faculdade de Odontologia, Departamento de Estomatologia, Disciplina de EstomatologiaClínica, Av. Prof. Lineu Prestes, 2227, Cidade Universitária, São Paulo, SP-Brazil, 05508-000; Tel and Fax: +55-11-38641372; E-mail: damiglia@usp.br

\begin{abstract}
Oral Lichenoid Contact Reaction (OLCR) mainly develops in response to amalgam filling. Since OLCR lesions cannot be distinguished either clinically or histopathologically from those of idiopathic oral lichen planus, the diagnosis of a suggestive OLCR can only be fully confirmed by observing the disappearance or a substantial improvement of the mucosal lesion after amalgam replacement. OLCR lesions are subtly symptomatic or even symptomless with many of them being diagnosed during routine dental examination. This article describes a case in which a forty-six old female patient was having a distressing pain related to OLCR, posing great difficulty for a consistent diagnosis. Following some unsuccessful attempted to treat the lesion with topical medication, the attention turned to amalgam replacement, resulting in the complete disappearance of the lesion.The relevance of this case is to remind clinicians that dealing witha difficult case paid off eventually, with an additional learning of an unusual and challenge clinical course of a simple oral condition.
\end{abstract}

Keywords: Amalgam replacement; Differential diagnosis; Oral Lichenoid Contact Reaction

\section{Introduction}

Oral Lichenoid Contact Reaction (OLCR) occurs mostly in association with amalgam filling. OLCR lesions mimic clinically and histologically those of idiopathic oral lichen planus, i.e., they can display the reticular, atrophic or erosive classic features seen in OLP $[1,2,3]$. A diagnosis of an OLCR lesion, therefore, should be guided by observing a direct contact or proximity of oral lesions to amalgam fillings followed by disappearance or improvement of lesions after amalgam replacement. Symptoms are not so a critical problem for patents with OLCR; usually they have mild complaints or no symptom at all [1,3].

A painful OLCR is quite unusual and may pose difficulties for its diagnosis. This article describes such a case in which the patient was experiencing a distressful pain.

\section{Case report}

A forty-six-year-old woman was referred to our clinic in July 2014 for evaluation of a painful lesion that had been present for 2 months. Oral examination revealed an isolated, shallow ulceration covered by whitish membrane interspersed with redness areas and whitish stria at its margin [Figure 1A].This lesion was causing her great difficulty on coping with her daily activities, mainly by impairing the ability on eating properly. Her medical history revealed that she had been treated for colon cancer a year ago, and was currently taking Levothyroxine (100 $\mathrm{mg}$ /daily). She was neither a smoker nor an alcohol drinker.

\section{Management}

The clinical diagnosis was of an erosive oral lichen planus lesion, erythema multiform and recurrent aphthous stomatitis. The latter was ruled out since there had not been any history of recurrences; the other clinical hypotheses were possible. Biopsy was not an initial option, since either diagnosis would be equally handled. For controlling her pain, an intralesional injection of triamcinolone was made and result was very successful, but the lesion recurred in 3 weeks in the same site causing the same symptoms. A new injection was made, to no avail. Searching for factors (such as medications, oral rinse, tooth paste or food) that could be accounted for the recalcitrant nature of the lesion, the only possible one was the amalgam restoration that was in contact with the lesion. After amalgam replacement, the patient experienced a total relief of symptoms as the lesion quickly disappeared, and has since been unchanged for 1.5 year [Figure 1B].

\section{Discussion}

Usually a diagnosis of OLCR is carried out without much difficulty. The suggestive cases are those when lesions exhibiting an OLP pattern are in direct contact or in close proximity with amalgam restorations [1,4]. Lesions showing this pattern will either disappear completely or improve substantially following amalgam replacement. If any change is not seen up to six weeks after treatment, then the diagnosis of an OLCR lesion shouldbe ruled out. A skin-patch test for mercury (the main component in amalgam feelings) may be of some help. But as it has been stated by most of authors, a negative patch-test result does not exclude a diagnosis of an OLCR lesion $[1,3,4,5]$. 
Under certain circumstances (such was the present case), a diagnosis of OLCR may delay because of some unusual presentation of the lesion. The presence of distressing pain is rarely associated with an OLCR lesion. And this fact weighed against to immediately replacing the amalgam filling. But later on, the amalgam replacement was the remaining possibility as the other attempts to control the pain had failed. Fortunately, it proved to be the right procedure. Another factor that could be associated with the lesion was the medication levothyroxine (a

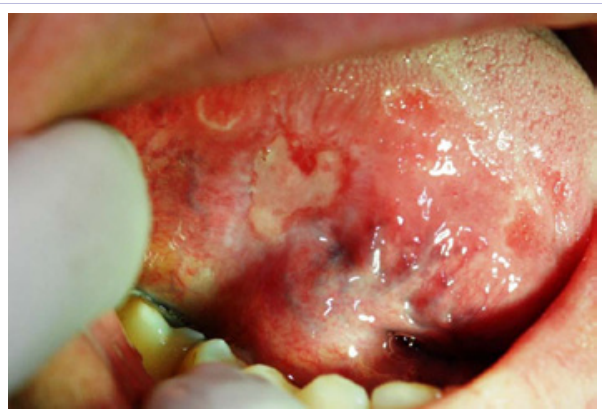

Figure 1a: This picture shows the ulceration and (discretely) an occlusal amalgam restoration on the tooth 46 . Concomitant with this lesion and occurring only by chance and not as part of this report, it is also possible to notice erythematous patches surrounded by serpiginous yellow striae, a characteristic feature of geographic tongue.

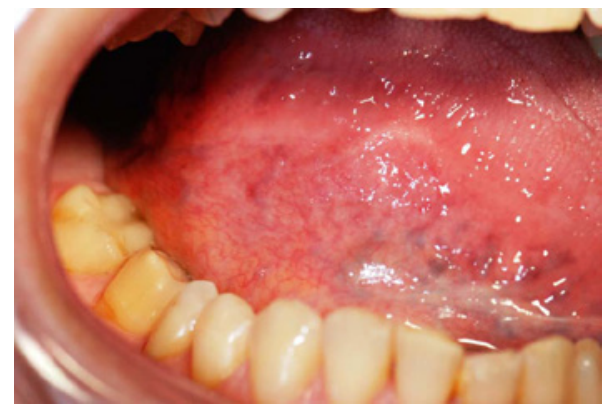

Figure 1b: The picture made after 1.5 follow-up. No signs of recurrence following amalgam replacement. hormone replacement for the treatment hypothyroidism) and, in such a case,the diagnosis would be switched to lichenoid drug reaction. But this possibility was ruled out since the patient was still under use of the medication long after the amalgam replacement had been made, and lesion did not recur.

Adding to the relevance of the present was to differentiate the idiopathic presentation of OLP from its counterpart OLCR, for two reasons. First, OLCR improves or even disappears after amalgam replacement; second, while idiopathic OLP may undergo malignant transformation, OLCR has not been associated with such a risk $[2,4,6]$. This was of the utmost importance for the patient since she had experienced a diagnosis and treatment of cancer in the intestine.

\section{Conclusion}

This case may add new insight on OLCR clinical course, as distressing pain can be part of its manifestation, therefore updating clinicians on this possibility.

\section{References}

1. Luiz AC, Hirota SK, Dal Vechio A, Reis VM, Spina R, Migliari DA. Diagnosing oral lichenoid contact reaction: clinical judgment versus skin-patch test. Minerva Stomatol. 2012;61(7-8):311-317.

2. van der Waal I. Oral lichen planus and oral lichenoid lesions; a critical appraisal with emphasis on the diagnostic aspects.Med Oral Patol Oral Cir Bucal. 2009;14(7):E310-E314.

3. Thornhill MH, Pemberton MN, Simmons RK, Theaker ED. Amalgamcontact hypersensitivity lesions and oral lichen planus. Oral Surg Oral Med Oral Pathol Oral RadiolEndod. 2003;95(3):291-299.

4. Issa Y, Brunton PA, Glenny AM, Duxbury AJ. Healing of oral lichenoid lesions after replacing amalgam restorations: A sistematic review. Oral Surg Oral Med Oral Pathol. 2004;98(5):553-565.

5. VG Suter, Warnakulasuriya S. The role of patch testing in the management of oral lichenoid reactions. J Oral Pathol Med. 2016;45(1):48-57.

6. Mattsson U, Jontell M, Holmstrup P. Oral lichen planus and malignant transformation: Is a recall of patients justified? Crit Rev Oral Biol Med. 2002;13(5):390-396. 